\title{
Digitale Publikations- und Forschungsinfrastrukturen
}

\author{
Niels Taubert
}

Abstract

Der Beitrag gibt eine Übersicht über zentrale Merkmale und Problemstellungen, die mit elektronischen Infrastrukturen in der Wissenschaft verbunden sind. Nach Bestimmung zentraler Merkmale digitaler Technologien fokussiert er auf zwei Typen. Für Forschungsdateninfrastrukturen werden die mit ihr verbundenen Zielsetzungen sowie Voraussetzungen einer Nutzung dargestellt. Für digitale Publikationsinfrastrukturen werden die typischen Produktions- und Verbreitungswege und die Forderung nach einem offenen Zugang zu Publikationen (Open Access) diskutiert. Dies mündet in die Darstellung spezifischer Problemstellungen, die sich im Zusammenhang mit digitalen Infrastrukturen ergeben.

\section{Keywords}

Wissenschaftliches Kommunikationssystem, Publikationssystem, Publikationsinfrastruktur, Forschungsdateninfrastruktur, Open Access, Open Data, Wissenschaftsverlag, Bibliothek, Publikation, Forschungsdaten

\section{Einleitung}

Ebenso wie andere Teilbereiche der Gesellschaft verändert sich die Wissenschaft aufgrund von Digitalisierungsschüben. In vielen Bereichen ist sie bereits heute digitalisiert, also von elektronischen Informations- und Kommunikationstechnologien durchdrungen, in anderen Teilen findet eine Aneignung dieser Möglichkeiten nur zögerlich und mit ungewissem Ausgang statt. Eine Vielzahl von Strukturen und Prozessen der Wissenschaft basieren bereits auf digitalen Technologien, greifen auf deren Ressourcen zurück oder werden von ihnen unterstützt: Experimental- oder Beobachtungsdaten werden, zum Teil unter Zuhilfenahme von Rechnerverbünden, in zunehmendem Umfang unmittelbar digital erfasst, im Verlauf des weiteren Forschungsprozesses digitalisiert und $\mathrm{zu}$ großen Datensammlungen zusammengeführt. Forschungsaktivitäten finden häufig kollaborativ und räumlich verteilt statt, die Auswertung von Forschungsdaten schließt oftmals die Nutzung elektronischer Verfahren und digitaler Technologien mit ein. Texte, die Forschungsergebnisse der 
wissenschaftlichen Community mitteilen, werden nahezu ausschließlich an Computern verfasst und im Zuge der Textproduktion wird auf digitale, online verfügbare Wissensbestände zurückgegriffen. Die Begutachtung, Publikation und Rezeption von Forschungsergebnissen erfolgt bereits heute meist auf der Grundlage digitaler Formate. Zur Vernetzung, zum informellen Austausch oder zur Information über die in der Community stattfindenden Aktivitäten wie beispielsweise Tagungen, Workshops und Konferenzen werden Web-2.0-Plattformen genutzt. Schließlich werden auch die Forschungsergebnisse über die Grenzen der Wissenschaft hinaus mithilfe elektronischer Medien verschiedenen wissenschaftlich interessierten Publika vermittelt. Beispiele sind Wissenschaftsblogs, Twitter oder Microblogs (siehe zur Übersicht Bonfadelli et al. 2016). Diese Omnipräsenz digitaler Technologien hat einige Beobachter der Entwicklung dazu veranlasst, zeitdiagnostisch von einem neuen Typus von Wissenschaft zu sprechen, der entweder als Cyberscience (2.0) (Nentwich 2003; Nentwich and König 2012), e-Science (Wouters 2006) oder e-Research (Jankowski 2007, 2009; Beaulieu and Wouters 2009) bezeichnet wird. Zu berücksichtigen ist bei einer solchen Diagnose allerdings das hohe Ausmaß an Uneinheitlichkeit der Adaption zwischen verschiedenen Fächern und Forschungsgebieten sowie die fortgesetzt hohe Innovationsdynamik digitaler Technologien, die darauf hindeuten, dass die Entwicklung weder zu einem Abschluss gekommen ist, noch sich entlang eines einheitlichen Pfads vollzieht.

Der vorliegende Beitrag gibt eine Übersicht über die digitale Produktion und Distribution von wissenschaftlichem Wissen. Um angesichts der vielfältigen Einsatzbereiche digitaler Technologien zu einem handhabbaren Zuschnitt zu gelangen, werden zwei Einschränkungen vorgenommen: Thematisch beschränkt sich der Beitrag auf zwei Anwendungsfelder. Im Bereich der Wissensproduktion fokussiert er auf Forschungsdaten, im Bereich der Wissensdistribution auf Publikationen. Andere, einleitend angedeutete Einsatzfelder bleiben dagegen ausgeklammert. In systematischer Hinsicht richtet er seinen Blick ausschließlich auf einen bestimmten Typus digitaler Technologien, nämlich auf solche mit Infrastrukturcharakter. Diese Akzentuierung erfolgt zum einen aufgrund des herausgehobenen Stellenwerts dieses Typs für die Wissenschaft und zum anderen mit Blick auf die spezifischen, mit ihm verbundenen wissenschaftspolitischen Problemstellungen.

In einem ersten Schritt wird der Charakter rezenter digitaler Technologien mithilfe des Begriffs der Infrastruktur bestimmt und die Begriffe Publikationsinfrastruktur und Forschungsdateninfrastruktur abgegrenzt. Im Anschluss daran wird in einem zweiten Schritt 
eine Übersicht über die Forschungsdateninfrastruktur gegeben, die mit ihr verbundenen Zielsetzungen dargestellt und die Voraussetzungen und Hindernisse einer Nutzung erörtert. Der dritte Schritt beschäftigt sich mit der digitalen Publikationsinfrastruktur, der für Publikationen typischen Produktions- und Verbreitungswege und mit der Forderung nach einem offenen Zugang zu elektronischen Publikationen (Open Access). Quer zu den beiden Bereichen werden in einem vierten Schritt einige spezifische wissenschaftspolitische Problemstellungen herausgearbeitet, die sich im Zuge des Aufbaus und Betriebs digitaler Infrastrukturen stellen. Der Beitrag schließt mit Forschungsdesideraten hinsichtlich Infrastrukturgenese und Wirkungen von Informationsinfrastrukturen.

\section{Digitale Infrastrukturen in der Wissenschaft}

Sowohl in der Forschungsliteratur als auch in der wissenschaftspolitischen Diskussion wird als Merkmal der derzeit in der Wissenschaft eingesetzten digitalen Technologien ihr Infrastrukturcharakter hervorgehoben. Beispielhaft sei hier der einflussreiche, von der National Science Foundation (NSF) herausgegebene Bericht Revolutionizing Science and Engineering Through Cyberinfrastructure (Atkins et al. 2003) genannt. Betont wird mit dieser Charakterisierung, dass die digitalen Technologien nicht nur gleich eines Werkzeugs situativ oder lokal wirksam sind, sondern in einem übergreifenden Kontext von einer Vielzahl von Akteuren genutzt werden. Dadurch prägen sie gleichzeitig an vielen Orten und unter den dort jeweils gegebenen Rahmenbedingungen die Wissenschaft.

Welche Merkmale sind darüber hinaus gemeint, wenn von Infrastruktur gesprochen wird? Aufgrund der Schwierigkeiten, Infrastrukturen mittels klarer, objektivierbarer Kriterien abzugrenzen, wird hier davon ausgegangen, dass der Begriff kontextabhängig ist und daher nur mit Blick auf einen bestimmten Bereich des Sozialen von ,Infrastruktur ${ }^{`}$ gesprochen werden kann. Mit diesem relationalen Verständnis wird die Frage, was Infrastrukturen im Kern ,sind“ und welche Komponenten zu ihr zählen, unterlaufen (Ribes and Lee 2010, p. 234). Infrastrukturen sind eingebettet in bestimmte Praxen oder Handlungszusammenhänge und bilden Arrangements aus technischen, sozialen und organisationalen Komponenten. Bei ihrer Verwendung zeichnen sie sich durch Transparenz aus, indem sie die Erledigung von Aufgaben unterstützen, dabei aber in den Hintergrund treten. Sie müssen nicht für jede Verwendung neu erfunden werden, sondern reichen über einzelne Nutzungsvorgänge und lokale Verwendungszusammenhänge hinaus. Charakteristisch ist ihre wechselseitige 
Verknüpfung mit Konventionen, indem sie selbst Konventionen der Verwendung prägen und ihrerseits von Konventionen geformt sind. Zudem sind sie über standardisierte Schnittstellen mit anderen Infrastrukturen verbunden. Eine Aufhebung der Transparenz findet statt, wenn es $\mathrm{zu}$ Fehlfunktionen kommt, sie also nicht in der Lage sind, Praxen und Handlungszusammenhänge effektiv zu unterstützen (Star and Ruhleder 1996, p. 113; Star 1999, p. 382; Karasti et al. 2010, pp. 382-383). Zu solchen Gelegenheiten wird deutlich, dass Infrastrukturen neben den Handlungszusammenhängen, in die sie eingebettet sind, noch über einen zweiten Umweltbezug verfügen. Sie sind ihrerseits von der Leistung von Organisationen abhängig, die die Wartung und Aufrechterhaltung im Zuge der Nutzung und Anpassung an veränderte Anforderungen übernehmen (Hughes 1987, p. 53).

Mit diesen knappen Erläuterungen zum Infrastrukturbegriff wird deutlich, dass digitale Wissenschaft drei analytisch zu unterscheidende Dimensionen mit sich führt: (a) das jeweilige soziale Handeln oder das Handlungssystem, in das die Infrastruktur eingebettet ist, (b) die Informationsinfrastruktur selbst, die für das Handlungssystem spezifische digitale Ressourcen bereitstellt sowie (c) Organisationen, die für die Aufrechterhaltung, Wartung und Entwicklung der Infrastruktur zuständig sind.

\section{Typen digitaler Informationsinfrastrukturen}

Das Feld der digitalen Wissenschaft lässt sich weiter entfalten, indem man die Handlungssysteme spezifiziert, für die Informationsinfrastrukturen Ressourcen bereitstellen. Quer zur Differenzierung verschiedener Disziplinen, Fächer und Forschungsgebiete (Stichweh 1979, 1992) verläuft die Unterscheidung zwischen dem Forschungssystem und dem formalen Kommunikationssystem. Innerhalb des Forschungssystems werden Wahrheitsansprüche formuliert, empirisch überprüft, getestet und verfeinert. Vorherrschend ist dort eine informelle Form wissenschaftlicher Kommunikation. Im formalen wissenschaftlichen Kommunikationssystem dagegen (Gravey and Griffith 1967; Whitley 1968) werden Wahrheitsansprüche geprüft, veröffentlicht, verbreitet, rezipiert und gegebenenfalls auch kritisiert. Neben diesen beiden zentralen Bereichen wird in der Literatur zum Teil noch ein dritter Bereich ausgemacht, der einen Transfer von wissenschaftlichem Wissen in andere gesellschaftliche Bereiche leistet (Nentwich 1999). Nutzt man diese Unterscheidungen zur Einordnung, lässt sich die digitale Informationsinfrastruktur des Forschungssystems als digitale Forschungsinfrastruktur und die des formalen 
Kommunikationssystems als digitale Publikationsinfrastruktur bezeichnen. Beide Infrastrukturen haben nicht-digitale Vorgänger.

Abbildung 1 illustriert den doppelten Umweltbezug digitaler Informationsinfrastrukturen in der Wissenschaft, sowie die Beziehungen zwischen den drei analytisch unterscheidbaren Dimensionen.

Abbildung 1: Informationsinfrastrukturen in der Wissenschaft

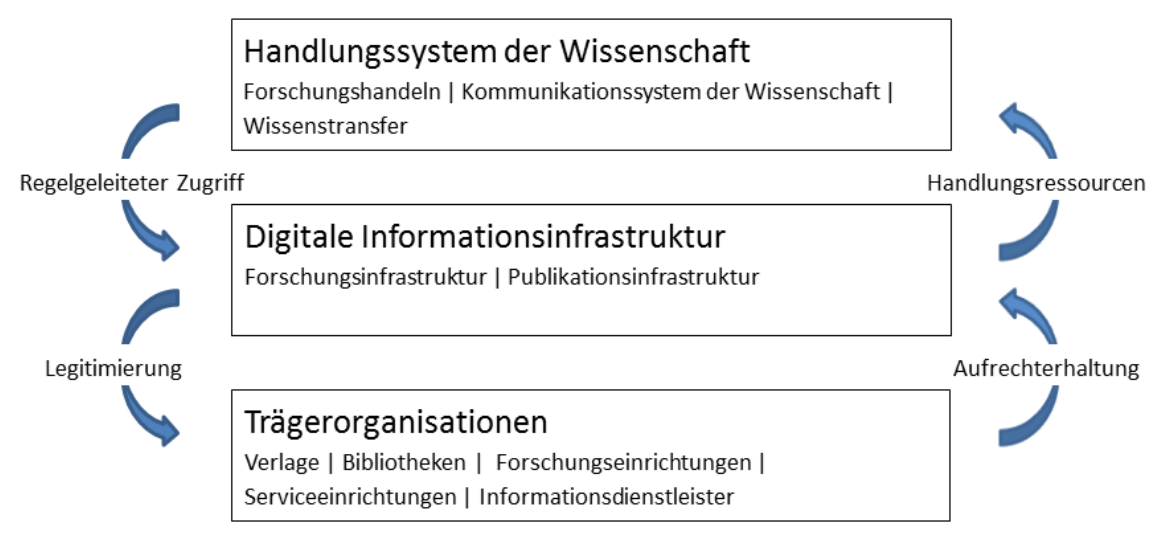

Quelle: eigene Darstellung

Abschließend soll auf zwei weitere Merkmale hingewiesen werden: Erstens sind digitale Informationsinfrastrukturen in der Wissenschaft prinzipiell kein neues Phänomen. Die ersten Anwendungen von Großrechnern fanden im Militär und in der Wissenschaft statt und auch die Netzwerktechnik, auf der das Internet basiert, hat ihre Ursprünge in diesen beiden Sozialsystemen (Campbell-Kelly and Aspray 1996, p. 87-99, 288-294). Es ist Kennzeichen der digitalen Informationsinfrastruktur (und dies unterscheidet sie mindestens graduell von anderen Infrastrukturen), dass sie fortwährend verändert, modifiziert und ergänzt wird. Daher lässt sich seit ihrem Auftreten bis in die Gegenwart hinein ein fortgesetzter Wandel beobachten, bei dem Wellen digitaler Technologien die Wissenschaft durchlaufen, dort Forschungs-, Publikations- und Rezeptionsroutinen modifizieren, neue Erwartungen und Anforderungen hervorrufen, die dann ihrerseits in die Weiterentwicklung der Informationsinfrastruktur münden. Zweitens werden mit dieser abstrakten Beschreibung universelle Merkmale der Digitalität erfasst. Eine Ebene darunter offenbart sich in verschiedenen Fächern und Forschungsgebieten ein hohes Maß an empirischer Diversität. Diese gilt für sämtliche der genannten drei Dimensionen also für die Erscheinungsform 
digitaler Informationsinfrastrukturen, bezüglich der Art und des Umfangs ihrer Nutzung und mit Blick auf die Merkmale der für die Aufrechterhaltung und Entwicklung zuständigen Trägerorganisationen.

\section{Digitale Wissensproduktion}

Das Forschungssystem der Wissenschaft, verstanden als all diejenigen Aktivitäten, die auf die Entwicklung, Formulierung und (empirische) Überprüfung von Wahrheitsansprüchen zielen, ist überaus heterogen. Neben verschiedenen Paradigmen oder Schulen sind es vor allem die vom Forschungsgegenstand gesetzten Rahmenbedingungen, die für Diversität sorgen: Forschungshandeln findet experimentierend oder beobachtend statt, arbeitet mit selbsterzeugten oder vorgefundenen, qualitativen oder quantitativen Daten und folgt einer mathematisierend-erklärenden oder einer sinnverstehenden Methodologie. Diese damit lediglich angedeutete Vielfalt führt dazu, dass digitale Wissensproduktion in verschiedenen Fächern und Forschungsbereichen unterschiedliche Formen hat und die eingesetzten digitalen Technologien sehr disparat sind.

Das Feld erschließt sich durch den Begriff der Forschungsinfrastruktur, worunter „diejenigen teilweise einzigartigen ,Einrichtungen, Ressourcen und Dienstleistungen“ in öffentlicher oder privater Trägerschaft verstanden [werden], die speziell für wissenschaftliche Zwecke errichtet, mittelfristig bis tendenziell permanent bereitgestellt werden und für deren sachgerechte Einrichtung, Betrieb und Nutzung in der Regel spezifische fachwissenschaftliche oder interdisziplinäre (Methoden-)Kompetenzen erforderlich sind. Ihre Funktion ist es, Forschung, Lehre und Nachwuchsförderung $\mathrm{zu}$ ermöglichen oder $\mathrm{zu}$ erleichtern [...] Sie werden nicht ausschließlich von einzelnen Personen oder Gruppen genutzt, sondern stehen prinzipiell einer internationalen Fachgemeinschaft oder mehreren Fachgemeinschaften offen“" (Wissenschaftsrat 2011a, S. 17). Der Begriff der Forschungsinfrastruktur ist offensichtlich breiter als der hier interessierende Gegenstand, da er auch nicht digitale Infrastrukturen wie Teilchenbeschleuniger, Teleskope, Forschungsschiffe und -flugzeuge mit einschließt. Die Definition ist aber dennoch instruktiv, weil sie Zwecksetzung, Trägerschaft, Kompetenz als Voraussetzung der Nutzung, Funktion und avisierte Nutzergruppe als wesentliche Merkmale nennt. 
Die folgende Darstellung berücksichtigt weder nicht-digitale Forschungsinfrastrukturen noch informationstechnische Infrastrukturen. Vielmehr bezieht sie sich auf einen spezifischen und in der derzeitigen Wissenschaftspolitik zentralen Typus der Forschungsinfrastruktur: die digitale Forschungsdateninfrastruktur. Mir ihr werden zu Zwecken der Forschung erhobene Daten gesammelt, organisiert und zur weiteren Nutzung bereitgehalten. Beispiele sind die Datenbank Simbad mit Beobachtungsdaten der Astronomie (Wenger et al. 2000), die Gendatenbank GenBank (Benson et al. 2011), das Tierstimmenarchiv (Deutsches Museum für Naturkunde o. Jg.), das Sozioökonomische Panel (Deutsches Institut für Wirtschaftsforschung o. Jg.) und die Datenbank der Deutschen digitalen Bibliothek mit einer Vielzahl von Digitalisaten (Deutsche digitale Bibliothek, o. Jg.).

Mit Blick auf das Forschungssystem dienen Forschungsdateninfrastrukturen vor allem drei Zwecken:

Wiederverwendung: Erstens verspricht die digitale Sammlung und Archivierung von Forschungsdaten ein höheres Maß an Effizienz, wenn mit öffentlichen Mitteln erhobene Daten im Rahmen neuer Fragestellungen nachgenutzt werden können. Ein solches Recycling ist vor allem in Forschungsgebieten von Nutzen, in denen das methodische Vorgehen weitgehend standardisiert ist und ähnliche Daten genutzt werden. Eine Nachnutzung von Forschungsdaten ist allerdings voraussetzungsvoll. Sie ist nur möglich, wenn der Datensatz und sein Zustandekommen (u.a. Erhebungsmethoden) dokumentiert sind. Bei einer kombinierenden Nachnutzung mehrerer Datensätze ist zudem auch eine Standardisierung technischer Formate und der Datenstruktur notwendig, damit eine Zusammenführung möglich ist.

Kreativfunktion: Generell liegt die Leistung von Forschungsinformationsinfrastrukturen in der Unterstützung und Ermöglichung von Forschungsprozessen. Dies gilt auch für nicht digitale Infrastrukturen wie beispielsweise Archive und Museen, ohne die in bestimmten Fächern ein systematischer Erkenntnisgewinn kaum möglich wäre. Von digitalen Forschungsdateninfrastrukturen erwartet man, dass der Zugang zu unterschiedlichen Arten von Daten zu grundlegenden Veränderungen des Forschungsprozesses führt. Es entstehen neue Möglichkeitshorizonte, wenn Forschungsfragen nachgegangen wird, die ohne einen digitalen Zugang kaum zu stellen und nicht zu beantworten wären. Dies gilt zum Beispiel für die Erforschung von ortsverteilten und ansonsten nur sehr aufwändig zu erreichenden Objekten und Beständen, zwischen denen so Zusammenhänge sichtbar gemacht werden können (Wissenschaftsrat 2011b, S. 17). 
Transparenz: Eine dritte Funktion bezieht sich auf die Glaubwürdigkeit von Forschungsergebnissen. Die Archivierung von Forschungsdaten, die publizierten Forschungsergebnissen zugrunde liegen, kann zu einer Vergrößerung der Transparenz des Forschungsprozesses führen. Zum einen werden mit $\mathrm{ihr}$ Zwischenergebnisse eines Forschungsprozesses dokumentiert. Beim Vorliegen entsprechender Auswertungsinstrumente und Kompetenzen können zum anderen einzelne Schritte des Forschungsprozesses nachvollzogen werden. Dokumentation und partielle Überprüfung des Zustandekommens der Ergebnisse können gleichermaßen dazu beitragen, das Vertrauen in Forschungsergebnisse zu stärken. Von Bedeutung ist diese Funktion insbesondere in Fächern, in denen es in der Vergangenheit zur Erosion von Vertrauen gekommen ist (Doorn et al. 2013; Franzen et al. 2007).

\section{Förderung der Datenarchivierung}

Angesichts des Nutzens, den die Forschungsdateninfrastruktur und Datenarchivierung im Grundsatz erbringen kann, stellt sich die wissenschaftspolitische Frage, durch welche Mechanismen die Nutzung der Archive zur Datenpublikation befördert werden kann. Ein grundsätzliches dabei zu lösendes Problem besteht darin, die Interessen der Datenproduzenten mit denen der Datennutzer in Ausgleich zu bringen.

Ein Anreiz für eine Datenpublikation besteht dann, wenn innerhalb einer Fachgemeinschaft Konventionen bestehen, die Weiterverwendung durch Zitation des Datensatzes kenntlich zu machen. Gut eingespielte Konventionen bestehen beispielsweise bereits seit längerer Zeit in der Astronomie. Ein Ausgleich zwischen den Interessen des Urhebers und des Nutzers kann dabei durch eine Sperrfrist für die Erstverwertung erfolgen (Ludwig und Enke 2013, S. 25). Ein Instrument, das Praktiken der Datenarchivierung und -nutzung stärker kodifiziert, sind so genannte Forschungsdaten-Policies von Universitäten und Forschungseinrichtungen (z.B. Universität Heidelberg, o. Jg.). Sie richten sich an ihre Angehörigen und ermuntern sie dazu, ihre Daten über Forschungsdatenrepositorien der Einrichtungen zugänglich zu machen. Während dieses Instrument lediglich eine Erwartung adressiert, die Entscheidung über die Archivierung von Forschungsdaten aber beim betreffenden Wissenschaftler belässt, finden sich daneben auch Regelungen mit stärker verpflichtendem Charakter. In diesen Bereich fallen die „Erwartung“ der Veröffentlichung von Forschungsdaten aus geförderten Projekten (z.B. Wellcome Trust 2010) und Vorschriften von Drittmittelgebern, der Beantragung von 
Drittmitteln Pläne zur Archivierung von Forschungsdaten beizufügen (z.B. NIH 2003). Eine harte Verpflichtung zur Archivierung von Forschungsdaten kann durch Rechtsnormen gegeben sein. Beispiele sind in Deutschland beispielsweise die Gentechnikaufzeichnungsverordnung (GenTAufzV), die Röntgenverordnung (RöV) und die Strahlenschutzverordnung (Ludwig und Enke 2013, S. 26).

Neben den Instrumenten, die auf eine Förderung der Datenarchivierung zielen, soll aber auch auf Hindernisse hingewiesen werden, die einer Veröffentlichung von Forschungsdaten entgegenstehen können. Neben nicht gegebenen technischen Voraussetzungen (wie der Verfügbarkeit entsprechender Datenrepositorien), Problemen auf der Ebene der Trägerorganisationen, der Befürchtung einer missbräuchlichen Verwendung von Forschungsdaten durch Nachnutzer (Parse 2009, pp. 25-26) und Interessen an der Sicherung der Exklusivität des Datenzugangs (Doorn et al. 2013, pp. 238-239) sind Datenschutzprobleme im Fall personenbezogener Daten von Bedeutung (z.B. Reichertz 2015). Diese treten insbesondere in der Medizin, der Psychologie und der Soziologie auf. Während in der quantitativen Sozialforschung praktische Wege der Anonymisierung und der Einholung des Einverständnisses der Befragten gefunden wurden und der Aufbau der digitalen Forschungsdateninfrastruktur im Bereich von Panel-Datensätzen und der amtlichen Statistik weit fortgeschritten sind (Wissenschaftsrat 2011b, S. 14), ist die einfache Übertragung auf den Bereich der qualitativen Forschung zum Teil mit Hindernissen verbunden. Die Anonymisierung kann die Verwertbarkeit von Daten für die weitere Forschung teils einschränken und die Einholung einer Einwilligung zur Datenarchivierung kann insbesondere bei sensiblen Forschungsgegenständen wie sozial unerwünschtem Verhalten unter Umständen das Vertrauensverhältnis zwischen Forscher und Informant beschädigen (Hirschauer 2014, S. 309). Daher muss die Frage nach dem Verhältnis von Nutzen, Schaden und Aufwand je nach Forschungsfeld und Datentypus differenziert beantwortet werden. Weitere Grenzen können der Archivierung durch einen urheberrechtlichen Schutz von Daten gesetzt oder auch ethisch begründet sein. Ein Beispiel dafür sind Forschungsergebnisse mit Dual-Use-Potential wie zum Beispiel Forschung mit hochpathogenen Mikroorganismen und denkbarer Anwendungsmöglichkeit als Biowaffe. 


\section{Freie Zugänglichkeit zu Forschungsdaten}

Die Frage, in welchem Umfang der oben beschriebene Nutzen der Archivierung von Forschungsdaten tatsächlich realisiert wird, hängt neben den gerade erwähnten Rahmenbedingungen auch von der Zugänglichkeit und Nutzbarkeit archivierter Daten ab. Innovative Forschungsideen können nur entwickelt werden, wenn die Daten und ihre Merkmale in Augenschein genommen werden können, eine Vergrößerung des Vertrauens in Forschungsergebnisse kann nur erreicht werden, wenn ein offener Zugang zu Primärdaten besteht und eine Nachnutzung ist nur möglich, wenn dem Zweitnutzer entsprechende Rechte eingeräumt werden. Daher setzt sich analog zur Forderung nach Open Access zu Publikationen (siehe Abschnitt 3) eine Vielzahl von Forschungseinrichtungen und Förderorganisationen für einen offenen Zugang und eine umfassende Nutzbarkeit von Daten zu Zwecken der Forschung ein. Eine einflussreiche Formulierung findet sich in der bereits im Jahr 2003 verabschiedeten Berlin Declaration:

„Open access contributions include original scientific research results, raw data and metadata, source materials, digital representations of pictorial and graphical materials and scholarly multimedia material. [...] The author(s) and right holder(s) of such contributions grant(s) to all users a free, irrevocable, worldwide, right of access to, and a license to copy, use, distribute, transmit and display the work publicly and to make and distribute derivative works, in any digital medium for any responsible purpose, subject to proper attribution of authorship" (Berlin Declaration 2003).

Dieses Ziel ist seit dieser Erklärung vielfach bekräftigt worden (z.B. Eurohorcs and ESF 2008) und hat national wie international zu einer Vielzahl von Aktivitäten geführt, so beispielsweise zum Open Research Data Programm des Förderprogramms Horizon 2020 der Europäischen Union (European Commission 2013, pp. 8-11).

\section{Digitale Wissensdistribution}

Die Distribution von neuen Wahrheitsansprüchen innerhalb wissenschaftlicher Gemeinschaften wird vom formalen Kommunikationssystem geleistet. Die für das System typische Kommunikation findet hier in Form der Publikation statt, die sich durch Schriftlichkeit und Öffentlichkeit auszeichnet und sich an einen nicht spezifizierten, potentiell unbeschränkten Adressatenkreis richtet. Zudem geht ihr ein förmlicher Akt der 
Veröffentlichung voraus und sie ist selbstreferentiell, indem Publikationen immer auch aus anderen Publikationen hervorgehen (Schimank 2012, S. 116). Sichtbar wird die Selbstbezüglichkeit durch Zitate, mit denen sich Publikationen aufeinander beziehen. Deren Besonderheit besteht in ihrem hohen Maß an Explizitheit, das es erlaubt, den Verweisen zu folgen und die Bezüge zwischen Wahrheitsansprüchen zu rekonstruieren. Ebenso wie das Forschungssystem weist auch das formale Kommunikationssystem erhebliche Unterschiede zwischen Fächer und Forschungsgebieten auf (siehe zusammenfassend: Alexander von Humboldt-Stiftung 2009; Taubert und Schön 2014, S. 10-14; Nederhof 2006). Trotz solcher Differenzen besteht in der Literatur Einigkeit darüber, dass das formale Kommunikationssystem über mehrere Unterfunktionen verfügt: Registrierung, Zertifizierung, Verbreitung und Archivierung (Kircz and Roosendaal 1996, pp. 107-108; Andermann und Degkwitz 2004, S. 8; Hagenhoff et al. 2007, S. 8; Taubert 2016).

- Mit Registrierung ist die nachprüfbare Bestimmung des Zeitpunkts gemeint, zu dem ein Wahrheitsanspruch erhoben wird. Sie ist entscheidend für die Rekonstruktion des Wissensfortschritts in einem Feld und für die Zuordnung der Priorität von Wahrheitsansprüchen zu einer oder mehreren Personen.

- Zertifizierung meint die Anerkennung eines Beitrags als Teil eines gemeinsamen Wissensstands, häufig durch Begutachtung (Peer Review). Erst mit der Zertifizierung gilt ein Beitrag als von der wissenschaftlichen Gemeinschaft akzeptiert und in den Wissensbestand aufgenommen.

- Verbreitung bezeichnet die Herstellung von Reichweite innerhalb einer wissenschaftlichen Kommunikationsgemeinschaft. Die Referenz auf die Kommunikationsgemeinschaft spezifiziert die Öffentlichkeit von Publikationen. Der prinzipiell unbeschränkte Adressatenkreis wird dadurch eingeschränkt, dass das Verständnis einer Publikation ein hohes Maß an Fachkompetenz voraussetzt.

- Archivierung beschreibt die fortlaufende Sicherung eines Wissensbestands über längere Zeiträume, so dass an ihn zu einem späteren Zeitpunkt mit weiterer Forschung angeschlossen werden kann. Sie ist zudem Voraussetzung für die Beurteilung von Forschungsleistungen. 
Im Laufe der letzten 20 Jahre hat sich in vielen Bereichen der Wissenschaft die digitale Publikation durchgesetzt und die elektronischen Publikationsmedien bilden eine wesentliche Komponente der Publikationsinfrastruktur. Ebenso wie in anderen Bereichen, in denen ein digitaler Medienwandel stattfindet, lässt sich sowohl die Substitution vorhandener Medien als auch deren Ergänzung beobachten. Die Substitution gedruckter Medien durch digitale Pendants findet vor allem in den Natur-, Lebens- und Technikwissenschaften und dort im Bereich der Journale statt. Die Initiative ging hier zunächst von der wissenschaftlichen Community aus. Noch vor der Öffnung des Internet für eine kommerzielle Nutzung und der Veröffentlichung des WorldWideWeb-Standards durch die Forschungseinrichtung CERN entstanden die ersten elektronischen Journale (Suber 2009), die per Email verbreitet wurden. Insbesondere die im Bereich von Science, Technology und Medicine (STM) tätigen Großverlage griffen den Innovationsimpuls ab Mitte der 1990er Jahre auf und erweiterten ihr Geschäftsfeld um digitale Zeitschriften. Für deren Vertrieb bauten sie Online-Plattformen (z.B. SpringerLink, ScienceDirect (Elsevier) und Wiley Online Library) auf, digitalisierten häufig in Kooperation mit Bibliotheken die älteren Jahrgänge ihrer Zeitschriften und entwickelten entsprechende Geschäftsmodelle.

Diese Veränderungen der Publikationsinfrastruktur berühren auch die Ebene der Trägerorganisationen und hier an erster Stelle das Verhältnis zwischen Verlagen und Bibliotheken. Grund dafür ist zum einen, dass digitale Journale im Vergleich zur gedruckten Zeitschrift in vielfältigerer Weise kommodifiziert werden können (Hanekop und Wittke 2006, S. 203-204, 2013, S. 151). Sie werden nicht nur als einzelne Zeitschrift oder im Rahmen größerer Zeitschriftenpakete im Abonnement vertrieben (Subskriptionsmodell). Darüber hinaus kann auch der Zugriff auf einen einzelnen Beitrag, die aktuelle Ausgabe, Teile oder sogar das gesamte Archiv einer Zeitschrift oder mehrerer Zeitschriften die zwischen Bibliotheken und Verlagen gehandelte Ware bilden und auch die dabei übertragenen Nutzungsrechte können sich unterscheiden. Zum anderen verändert sich die Aufgabenverteilung zwischen Bibliotheken und Verlagen bei der Wahrnehmung der Verbreitungsfunktion. Während bei der gedruckten Publikation Verlage eine bestimmte Auflage eines Druckwerks produzieren, in Verkehr bringen und bewerben, organisieren Bibliotheken einen Verleihverkehr und vergrößern die Reichweite der Publikationen erheblich. Diese Aufteilung verschiebt sich bei der digitalen Publikation in Richtung der Verlage, da diese Wissenschaftler nunmehr direkt mit Publikationen versorgen. Bibliotheken büßen damit eine maßgebliche Funktion in der Vertriebskette ein, wenngleich lokale Gegebenheiten damit nicht unwichtig werden. Mit dem Fortschreiten der digitalen Publikation 
ist es zunehmend weniger von Bedeutung, wie groß der von einer Bibliothek gesammelte Literaturbestand vor Ort ist, während die Frage, welche Art und welcher Umfang an Lizenzen von einem bestimmten Ort den Zugriff auf global prinzipiell erreichbare, aber zugangsbeschränkte Bestände erlauben, stark an Relevanz gewinnt.

\section{Freie Zugänglichkeit zu Publikationen}

Eine zweite Entwicklung ist mit der digitalen Publikation eng verknüpft, da sie diese zur Voraussetzung hat: Open Access, womit der leserseitig freie Zugang zu elektronischen Publikationen gemeint ist. Dieser Zusammenhang wird bereits im Jahr 2002 in einer einflussreichen Erklärung treffend benannt:

„An old tradition and a new technology have converged to make possible an unprecedented public good. The old tradition is the willingness of scientists and scholars to publish the fruits of their research in scholarly journals without payment, for the sake of inquiry and knowledge. The new technology is the internet. The public good they make possible is the world-wide electronic distribution of the peer-reviewed journal literature and completely free and unrestricted access to it by all scientists, scholars, teachers, students, and other curious minds" (Budapest Open Access Initiative 2002).

Veranlasst ist die Forderung eines uneingeschränkten Zugangs von mit öffentlichen Mitteln geförderter Forschungsliteratur unter anderem durch eine Krisensituation wissenschaftlicher Bibliotheken. Seit den 1980er Jahren haben sich wissenschaftliche Großverlage im Bereich von STM zunehmend an ökonomischen Renditezielen orientiert, was $\mathrm{zu}$ starken Preissteigerungen für Subskriptionen im Bereich der Journale geführt hat. Im Zeitraum 1975 bis 1995 erhöhten sich die Preise um zwei- bis dreihundert Prozent (European Commission 2006, p. 16), für 1986 bis 2006 werden jährliche Preissteigerungen von fünf bis acht Prozent (Kirchgässner 2008) und für den Zeitraum 2008 bis 2010 von zwischen sieben und zehn Prozent (Boni 2010) berichtet. Da die Menge an Forschungsliteratur fortwährend wächst, die Bibliotheksbudgets aber kein vergleichbares Wachstum verzeichnen, können die Preissteigerungen nicht aufgefangen werden und die Bibliotheken sind gezwungen, ihre Erwerbs- und Sammelaktivitäten einzuschränken. Dies betrifft auch den Bereich der Monographien (Kopp 2000).

Die Großverlage veränderten aufgrund der Forderung nach einer freien Zugänglichkeit ihre 
Geschäftsmodelle und griffen eine der im Text der Budapest Open Access Initiative erwähnten Option auf - die Herstellung freier Zugänglichkeit am originären Publikationsort, das so genannte Gold Open Access. Finanziert wird in diesem Modell die Publikation auf der Seite der Autoren meist durch Publikationsgebühren (auch Article Processing Charges, APC genannt), die nicht vom Autor selbst, sondern von der ihn beschäftigenden Organisation aus Publikationsfonds bezahlt werden (Arbeitsgruppe Open Access [... 2014).

Die Budapest Initiative on Open Access nennt daneben noch ein zweites Modell, das Green Open Access genannt wird. Seinen Ursprung hat es ebenfalls in der wissenschaftlichen Gemeinschaft. In der Physik wurde im Jahr 1991 ein Server (im Folgenden Repositorium) für die Ablage und Zugänglichmachung von Manuskripten eingerichtet, die bei Zeitschriften zur Publikation eingereicht wurden (Ginsparg 2011, p. 145). Dieses Repositorium arXiv knüpft lose an die Praxis der Zirkulation von Preprints, also noch nicht veröffentlichten Manuskripten, an, stellt aber eher eine Ergänzung als eine Substitution bestehender Publikationsmedien dar. Es schafft eine zweite Ebene der Publikationsinfrastruktur, die eine (frühzeitige) Zirkulation von Forschungsergebnissen ermöglicht und dem Autor durch einen Zeitstempel die Priorität an der Veröffentlichung sichert. Was die Begutachtung angeht, bleibt das Modell auf die Leistungen von Journalen angewiesen. Daher lässt sich sagen, dass durch das Green-Open-Access-Modell Verbreitungs- und Registrierungsfunktion einerseits und Zertifizierungsfunktion andererseits auseinandertreten und sich auf verschiedene Publikationsmedien verteilen. Mittlerweile reklamieren die Bibliotheken den Betrieb von Repositorien als Aufgabenfeld. Entstanden ist in den letzten Jahren eine beachtliche Repositorieninfrastruktur, die allein für Deutschland 177 Repositorien aufweist (OpenDOAR 2015).

\section{Reorganisation des Peer Review}

Mit der digitalen Publikationsinfrastruktur wandelt sich nicht nur das Publikationsmedium selbst, sondern auch die Art der Realisierung der Zertifizierungsfunktion (Peer Review) und die Produktion von Publikationen. Parallel $\mathrm{zu}$ den weiter oben erwähnten Publikationsplattformen, und technisch mit diesen durch Workflows verknüpft, haben Verlage so genannte Online-Editorial-Management-Systeme eingeführt und damit den Begutachtungsprozess sowie die Zusammenarbeit von Wissenschaftlern und Verlagen auf der Grundlage digitaler Informations- und Kommunikationstechnologien reorganisiert. Die Systeme bringen alle am Begutachtungs- und Produktionsprozess Beteiligten zusammen und 
organisieren Einreichung, Begutachtung und die technische Produktion über diese Plattform. Das Vorliegen sämtlicher Dokumente (Einreichung, Grafiken, Gutachten, Daten und Korrespondenz der Beteiligten) bildet die Grundlage zur Verlagerung von Arbeitsschritten häufig zu Lasten der beteiligten Wissenschaftler und für die Auslagerung von Arbeitsschritten innerhalb des Verlags in Niedriglohnländer (Taubert 2012). Diese Veränderungen sorgen zwar für eine Reorganisation des klassischen Peer-Review-Verfahrens, das aber in seiner Grundstruktur (Bestellung von zwei meist anonym bleibenden Gutachtern durch den Herausgeber) erhalten bleibt.

Tiefgreifendere Verfahrensinnovationen stellen dagegen Open Peer Review, Public Peer Review, Post Publication Peer Review und Open Discussion dar, die sich zwar bei einzelnen Journalen oder Publikationsplattformen haben etablieren können, derzeit aber keine Standardverfahren sind (Ware 2008, p. 18). Beispiele erfolgreicher Adaptionen dieser Modelle sind hier die Zeitschriften British Medical Journal (BMJ) und Atmospheric Chemistry and Physics sowie die Publikationsplattformen Faculty of 1000 (F1000) und ScienceOpen. Ohne die Verfahren im Einzelnen würdigen zu können, soll hier die Aufmerksamkeit darauf gerichtet werden, dass diese Modelle in mindestens dreierlei Hinsicht innovativ sind: Erstens weiten die Verfahren zum Teil den Kreis derjenigen aus, die an der Begutachtung mitwirken. Die Beteiligten werden nicht durch einen Herausgeber ausgewählt sondern beteiligen sich qua Selbstselektion. Zweitens wird das Redaktionsgeheimnis teils partiell, teils sogar vollständig aufgehoben, so dass die im Zuge der Begutachtung vorgebrachten Argumente geprüft werden können. Daneben kann zum Teil auch nachvollzogen werden, wer am Entscheidungsprozess mitgewirkt hat. Drittens findet die Auszeichnung von Qualität nicht vor Publikation eines Beitrags statt, sondern im Zuge der Rezeption nach dem Prinzip "publish then filter" (Hunter 2012, p. 2). Ohne Frage sind nicht sämtliche Verfahren für jedes Journal und für jedes Forschungsgebiet gleichermaßen geeignet. Interessant sind diese Entwicklungen dennoch, weil sie ein höheres Maß an Transparenz und Nachvollziehbarkeit des Begutachtungsverfahrens realisieren können.

Der derzeitige Stand der Entwicklung digitaler Publikationsinfrastrukturen lässt sich wie folgt zusammenfassen: Die digitale Publikation hat sich in vielen Fächern und Forschungsgebieten auf breiter Front durchgesetzt und dies gilt insbesondere dort, wo Journal-Artikel die dominierende Form der Publikation sind. Weniger stark verankert ist sie in Teilen der Geistes- und Sozialwissenschaften, in denen insbesondere bei der Rezeption längerer Texte eine Präferenz für gedruckte Texte fortbesteht. Hinzu kommt, dass die Verlagslandschaft kleinteiliger ist und aufgrund der geringeren finanziellen Leistungsfähigkeit kleiner Verlage 
die Entwicklung elektronischer Formate und Vertriebswege weniger weit fortgeschritten ist (Volkmann et al. 2014, S. 208).

Die Transformation in Richtung Open Access ist in einem größeren Umfang bisher nur in einzelnen Wissenschaftsbereichen gelungen. Gemessen am Volumen frei zugänglicher Publikationen stellt Green Open Access das bedeutendere Modell dar: Im Web of Knowledge ist im Publikationsjahrgang 2011 ein Anteil von 9,0 Prozent der Publikationen am originären Publikationsort frei zugänglich, in der Zitationsdatenbank Scopus liegt dieser Anteil bei 11 Prozent (Laakso and Björk 2012, p. 6). Der Anteil an Publikationen, die aus demselben Jahrgang in Repositorien im Green Open Access verfügbar sind liegt dagegen bei 21,9Prozent. Dabei schwankt er zwischen den Disziplinen erheblich. Das Schlusslicht nimmt die Chemie mit einem Anteil von 9,3 Prozent selbstarchivierter Publikationen ein, der größte Anteil findet sich in der Mathematik mit 40,8 Prozent (Gargouri et al. 2012, p. 8). Es ist bereits darauf hingewiesen worden, dass mit Repositorien eine zweite Publikationsebene geschaffen wurde, die die Zirkulation von Forschungsergebnissen unterstützt. Allerdings besteht häufig Unklarheit darüber, ob die dort abgelegte Version auch dem Wortlaut der Journalversion entspricht und durch Unterschiede im Layout (z.B. durch das Fehlen einer Paginierung) kann sie zum Teil nur eingeschränkt verwendbar sein. Daher bleibt allen Anstrengungen zum Trotz die Abhängigkeit vom originären Publikationsort, der in der Regel immer noch das subskriptionspflichtige Journal ist, bestehen und die Lizensierung entsprechender Angebote durch Forschungsförderorganisationen und Bibliotheken bildet einen zentralen Bestandteil der wissenschaftlichen Informationsversorgung.

\section{Problemstellungen der Wissenschaftspolitik}

Die Entwicklung von Publikations- und der Forschungsdateninfrastrukturen stellt eine große wissenschaftspolitische Herausforderung dar. Grund dafür ist nicht nur der hohe Ressourceneinsatz sondern auch das eingangs erwähnte Merkmal von Infrastrukturen, nicht nur lokal angemessene Lösungen bereitzustellen, sondern für ganze Fächer oder Forschungsgebiete nutzbar zu sein. Auf die Herausforderung der fortgesetzten Digitalisierung hat die Wissenschaftspolitik reagiert, indem sie durch die Abgrenzung verschiedener Handlungsfelder die Komplexität der Aufgabe kleinzuarbeiten sucht. Solche Felder sind: Retrodigitalisierung, Langzeitarchivierung, Lizensierung, Hosting lizensierter Inhalte, Virtuelle Forschungsumgebungen, Nicht-textuelle Materialien, Open Access, Forschungsdaten sowie Informationskompetenz/Ausbildung (Wissenschaftsrat 2011a; KII 
2011). Quer zu diesen Handlungsfeldern ergeben sich für die Akteure typische Problemstellungen, die im Zuge des Infrastrukturaufbaus bewältigt werden müssen.

Lokal gewachsene Strukturen und universelle Nutzung: Die Entwicklung der digitalen Infrastruktur in der Wissenschaft vollzieht sich typischerweise nicht zentral geplant, sondern geht häufig zunächst von lokalen, zum Teil auch durch die Laufzeit von Forschungsprojekten zeitlich begrenzten Anforderungen aus. Der infrastrukturelle Anspruch nach universeller Nutzbarkeit kann dazu in ein Spannungsverhältnis treten. Zur Erreichung dieses Ziels kann es notwendig sein, die Nutzungsmöglichkeiten einer lokalen Infrastruktur soweit zu skalieren, dass eine breitere Nutzung möglich ist oder verschiedene technische Lösungen soweit zu vereinheitlichen, dass sie als eine einheitliche Infrastruktur nutzbar werden. Insbesondere die Entwicklung einer Infrastruktur aus mehreren lokalen Ursprüngen erfordert einen hohen Koordinationsaufwand, wie beispielsweise die Entwicklung bibliothekarischer Nachweissysteme zeigt.

Finanzierung weltweit nutzbarer Infrastrukturen: Ein zweites, für die digitale Wissenschaft typisches Problem besteht in der Frage nach der Verteilung finanzieller Lasten der Einrichtung und des Betriebs digitaler Informationsinfrastrukturen. Welche Anreize existieren für eine Forschungseinrichtung, knappe finanzielle Mittel für Ressourcen aufzuwenden, die nicht nur lokal nutzbar sind? Sinnfälliges Beispiel ist die Umstellung von Journalen auf ein APC-finanziertes Open-Access-Modell und der dort anzutreffende first mover disadvantage. Diejenigen Forschungseinrichtungen, die sich zuerst bereiterklären, Publikationsgebühren für ihre Autoren zu übernehmen müssen zudem Mittel für die Subskription von Zeitschriften aufwenden, um den Zugang zu Forschungsbeiträgen herzustellen, die nicht im Open Access publiziert werden. Die Frage nach der Lastenverteilung wiederholt sich bei global zu nutzenden Infrastrukturen auf der Ebene der Bundesländer und zwischen Staaten.

Transfer von Lösungen: Drittens lässt sich eine Tendenz erkennen, in bestimmten Kontexten erfolgreiche Modelle von Infrastrukturen in andere Bereiche der Wissenschaft zu übertragen. Für den betroffenen Bereich kann sich dieser Transfer als coercive isomorphism (DiMaggio and Powell 1983, p. 150) darstellen, seitens der forschungsfördernden Organisationen vom Wunsch nach über möglichst universellen Strukturen motiviert sein. Angesichts der Diversität der Rahmenbedingungen in verschiedenen Fächern und Forschungsgebieten trifft dies auf eine unterschiedlich große Offenheit und Nutzungsbereitschaft gegenüber der angebotenen Infrastruktur, die nicht immer als nützlich wahrgenommen werden muss. Eine mangelnde Rückkopplung zwischen den Anforderungen der Wissenschaft und dem Infrastrukturaufbau 
kann zu einer eingeschränkten und hinter den Erwartungen zurückbleibenden Nutzung führen. Ein Beispiel dafür bilden Repositorien für Publikationen, die in einem stark unterschiedlichen Umfang genutzt werden.

Öffentliche versus privatwirtschaftliche Trägerschaft von Infrastrukturen: In seinen übergreifenden Empfehlungen zu Informationsinfrastrukturen in der Wissenschaft stellt der Wissenschaftsrat fest, die Gewährleistung der Verfügbarkeit von Informationsinfrastrukturen sei eine öffentliche Aufgabe (Wissenschaftsrat 2001a, S. 7). Diese Formulierung benennt zwar eine Zuständigkeit der öffentlichen Hand, lässt aber offen, wie diese wahrgenommen wird und ob und wenn ja, in welchem Umfang privatwirtschaftliche Akteure an der Trägerschaft von Infrastrukturen beteiligt sein sollen. Die sich stellende Aufgabe besteht darin, die Vorteile des öffentlichen und des privatwirtschaftlichen Organisationsmodells jeweils $\mathrm{zu}$ nutzen und gegebenenfalls in gelungener Weise $\mathrm{zu}$ kombinieren. Für Forschungsdateninfrastrukturen und Publikationsinfrastrukturen sind dabei die Ausgangslagen verschieden. Im Zusammenhang mit Open Access sind Bestrebungen zu erkennen, die Effekte einer privatwirtschaftlichen Trägerschaft hinsichtlich des Zugangs zu Publikationen und bezüglich der Kosten einzudämmen und die Infrastruktur stärker in die Verantwortung öffentlich finanzierter Einrichtungen zu überführen. Dies gelingt zwar im Bereich der Repositorien, hat im Bereich der Journale angesichts der Besitzverhältnisse aber Grenzen. Bei Forschungsdateninfrastrukturen liegt die Trägerschaft dagegen meist bei öffentlichen Einrichtungen. Hier stellt sich dagegen die Frage, ob und wenn ja, inwieweit eine privatwirtschaftliche Trägerschaft überhaupt denkbar ist.

\section{Fazit}

Der Beitrag hat eine Übersicht über Entwicklungen im Bereich der Publikations- und Forschungsdateninfrastrukturen gegeben und mithilfe der Unterscheidung von drei Ebenen verschiedene Dimensionen einer Wissenschaft ausgeleuchtet, die digitale Technologien intensiv nutzt. Dies lässt es zu, die verschiedenen Relationen zwischen dem Handlungssystem der Wissenschaft und der digitalen Infrastruktur einerseits und zwischen der Infrastruktur und ihren Trägerorganisationen andererseits $\mathrm{zu}$ fokussieren. Die dynamische Entwicklung digitaler Infrastrukturen innerhalb der Wissenschaft stellt nicht nur eine Herausforderung für die Wissenschaftspolitik dar, sondern auch für die diesen Prozess reflektierende Wissenschaftsforschung. Nach einer Phase weitgehender Abstinenz, in der der 
Forschungsgegenstand weitgehend der Informations- und Bibliothekswissenschaft überlassen wurde, lassen sich nun auch dort einige Bestrebungen erkennen, die mit diesem Wandlungsprozess verbundenen Veränderungen zu untersuchen. Angesichts der Breite des Wandlungsprozesses der digitalen Wissenschaft sind diese Anstrengungen als bescheiden zu bezeichnen. Forschungsbedarf besteht daher sowohl in Bezug auf die Infrastrukturgenese als auch mit Blick auf die transformierenden Wirkungen, die von Informationsinfrastrukturen auf die Wissenschaft ausgehen. Die Etablierung einer solchen Forschungstradition könnte nicht nur einen Beitrag zur Aufklärung der Dynamik des digitalen Wandels der Wissenschaft leisten, sondern für die Wissenschaftspolitik eine wertvolle Ressource für die Entwicklung von digitalen Informationsinfrastrukturen sein.

\section{Literatur}

Alexander von Humboldt Stiftung (Hrsg.). 2009. Publikationsverhalten in unterschiedlichen Disziplinen. Beiträge zur Beurteilung von Forschungsleistungen. 2. erw. Aufl. Bonn:

Diskussionspapier der Alexander von Humboldt-Stiftung 12/2009.

Andermann, Heike und Andreas Degkwitz. 2004. Neue Ansätze in der wissenschaftlichen Informationsversorgung. Ein Überblick über Initiativen und Unternehmungen auf dem Gebiet des elektronischen Publizierens. Historical Social Research 29(1): 6-55.

Arbeitsgruppe Open Access der Schwerpunktinitiative Digitale Information der Allianz der deutschen Wissenschaftsorganisationen (Hrsg.). 2014. Open-Access-Publikationsfonds. Eine Handreichung. http://doi.org/10.2312/allianzoa.006. Gesehen 01. November 2015.

Atkins, Daniels E., Kelvin K. Droegemeier, Stuart I. Feldmann, Hector Garcia-Molina, Michael L. Klein, David G. Messerschmitt, Paul Messina, Jeremiah P. Ostriker and Margaret H. Wright. 2003. Revolutionizing Science and Engineering Through Cyberinfrastructure: Report of the National Science Foundation Blue-Ribbon Advisory Panel on Cyberinfrastructure. http://www.nsf.gov/od/oci/reports/atkins.pdf. Gesehen 31. Oktober 2015.

Beaulieu, Anne and Paul Wouters. 2009. e-Research as Intervention. In e-Research. Transformation in Scholarly Practice. Hrsg. Nicholas W. Jankowski, 54-69. New York/London: Routledge. 
Benson, Dennis A., Ilene Karsch-Mizrachi, David J. Lipman, James Ostell and Eric W. Sayers. 2011. GenBank. Nucleic Acids Research, 2011(39): D32-D37.

Berlin Declaration on Open Access to Knowledge in the Sciences and Humanities. 2003. http://openaccess.mpg.de/Berliner-Erklaerung. Gesehen 02. November 2015.

Boni, Manfred. 2010. Analoges Geld für digitale Zeilen: der Publikationsmarkt der Wissenschaft. Leviathan 38(3): 293-312.

Bonfadelli, Heinz, Birte Fähnrich, Corinna Lüthje, Jutta Milde, Markus Rhomberg, und Mike Schäfer (Hrsg.). 2016. Forschungsfeld Wissenschaftskommunikation. Wiesbaden: VS-Verlag. Budapest Open Access Initiative. 2002. http://www.budapestopenaccessinitiative.org/read. Gesehen 01. November 2015.

Campbell-Kelly, Martin und William Aspray. 1996. Computer. A history of the information machine. New York: Basic Books.

Deutsche digitale Bibliothek. o. Jg. https://www.deutsche-digitale-bibliothek.de/. Gesehen 01. November 2015.

Deutsches Institut für Wirtschaftsforschung. o. Jg. Sozioökonomisches Panel. http://www.diw.de/soep. Gesehen 01. November 2015.

Deutsches Museum für Naturkunde. o. Jg. Tierstimmenarchiv. http://www.tierstimmenarchiv.de/. Gesehen 01. November 2015.

DiMaggio, Paul J. and Walter W. Powell. 1983. The Iron Cage Revisited: Institutional Isomorphism and Collective Rationality in Organizational Fields. American Sociological Review 48(2): 147-160.

Doorn, Peter, Ingrid Dillo and René van Horik. 2013. Lis, Damend Lis and Rearch Data: Can Data Sharing Prevent Data Fraud? The International Journal of Digital Curation 8(1): 229243.

Eurohorcs and ESF (European Science Fondation). 2008. Science Policy Briefing The EUROHORCs and ESF Vision on a Globally Competitive ERA and their Road Map for Actions to Help Build It. Juni 2008.

http://www.esf.org/fileadmin/Public_documents/Publications/SPB33_ERARoadMap.pdf. Gesehen 01. November 2015.

European Commission. 2006. Study on the economic and technical evolution of the scientific publication markets in Europe. Final Report January 2006. Brussels: European Commission, 
DG Research. http://ec.europa.eu/research/science-society/pdf/scientific-publicationstudy_en.pdf. Gesehen 01. November 2015.

European Commission. 2013. Guidelines on Open Access to Scientific Publications and Research Data in Horizon 2020.

http://ec.europa.eu/research/participants/data/ref/h2020/grants_manual/hi/oa_pilot/h2020-hioa-pilot-guide_en.pdf. Gesehen 02. November 2015.

Franzen, Martina, Simone Rödder und Peter Weingart. 2007. Fraud: causes and culprits as perceived by science and the media. EMBO-Reports 8(1): 3-7.

Gargouri, Yassine, Vincent Larivière, Yves Gingras, Les Carr and Stevan Harnad. 2012. Green and Gold Open Access Percentages and Growth, by Discipline". arXiv:1206.3664: (2012).

Ginsparg, Paul. 2011. ArXiv at 20. Nature 476 (11. August 2011): 145-147.

Gravey, William D. and Belver C. Griffith 1967. Scientific communication as a Social System. Science 157 (3762): 1011-1016.

Hagenhoff, Svenja, Lutz Seidenfaden, Björn Ortelbach und Matthias Schumann. 2007. Neue Formen der Wissenschaftskommunikation. Eine Fallstudienuntersuchung. Göttinger Schriften zur Internetforschung, Bd. 4. Göttingen: Göttinger Universitätsverlag.

Hanekop, Heidemarie und Volker Wittke. 2006. Das wissenschaftliche Journal und seine möglichen Alternativen: Veränderungen der Wissenschaftskommunikation durch das Internet. In Internetökonomie der Medienbranche, Göttinger Schriften zur Internetforschung, Hrsg. Svenja Hagenhoff, 201-233. Göttingen: Universitätsverlag Göttingen.

Hanekop, Heidemarie und Volker Wittke. 2013. Der Wandel des wissenschaftlichen Publikationssystems durch das Internet. Sektoriale Transformation im Kontext institutioneller Rekonfiguration. In Internet, Mobile Devices und die Transformation der Medien. Radikaler Wandel als schrittweise Rekonfiguration, Hrsg. Ulrich Dolata und Jan-Felix Schrape, 147172. Berlin: Edition Sigma.

Hirschauer, Stefan. 2014. Sinn im Archiv? Zum Verhältnis von Nutzen, Kosten und Risiken der Datenarchivierung. Soziologie 43(3): 300-312.

Hughes, Thomas P. 1987. The Evolution of Large Technological Systems. In The Social Construction of Technological Systems. New Directions in the Sociology and History of 
Technology, eds. Wiebe E. Bijker, Thomas P. Hughes and Trevor J. Pinch, 51-82. Cambridge MA: The MIT Press.

Hunter, Jane. 2012. Post-publication peer review: opening up scientific conversation. Frontiers in Computational Neuroscience. 6(63): 1-2.

Jankowski, Nicholas W. 2007. Exploring e-Science: An Introduction. Journal of ComputerMediated Communication 12: 549-562.

Jankowski, Nicholas W. 2009. The Contours and Challenges of e-Research. In e-Research. Transformation in Scholarly Practice, ed. Nicholas W. Jankowski, 3-33. New York/London: Routledge.

Karasti, Helena, Karen Baker and Florence Millerand. 2010. Infrastructure Time: Long-term Matters in Collaborative Development. Computer Supported Cooperative Work 19: 377-415. KII (Kommission Zukunft der Informationsinfrastruktur) 2011. Gesamtkonzept für die Informationsinfrastruktur in Deutschland. http://www.leibnizgemeinschaft.de/fileadmin/user_upload/downloads/Infrastruktur/KII_Gesamtkonzept.pdf. Gesehen 01. November 2015.

Kirchgässner, Adalbert. 2008. Zeitschriftenkonsortien. Angebotsausweitung auf Kosten der Flexibilität. In Informationskonzepte für die Zukunft. ODOK '07 (Schriften der Vereinigung Österreichischer Bibliothekarinnen und Bibliothekare 5), Hrsg. Eveline Pipp, S. 137-146. Graz-Feldkirch: Wolfgang Neugebauer Verlag GesmbH.

Kircz, Joost G. und Hans E. Roosendaal. 1996: Understanding and shaping scientific information transfer. In Electronic Publishing in Science, Proceedings of the Joint ISCU Press/UNESCO Expert Conference, eds. Dennis Shaw and Howard Moore, 106-116. Paris, 19.-23. 02. 1996.

Kopp, Hans 2000. Die Zeitschriftenkrise als Krise der Monographienbeschaffung. Bibliotheksdienst 34(11): 1822-1827.

Laakso, Mikael and Bo-Christer Björk. 2012. Anatomy of open access publishing: a study of longitudinal development and internal structure. BMC Medicine 10: 124.

Ludwig, Jens und Harry Enke (Hrsg.). 2013. Leitfaden zum Forschungsdaten-Management Handreichungen aus dem WissGrid-Projekt. Glückstadt: Verlag Werner Hülsbusch.

Nederhof, Anton J. 2006. Bibliometric monitoring of research performance in the Social Sciences and the Humanities: A review. Scientometrics 66(1): 81-100. 
Nentwich, Michael. 1999. Cyberscience: Die Zukunft der Wissenschaft im Zeitalter der Informations- und Kommunikationstechnologien. MPIfG Working Paper 99/6, Mai 1999.

Nentwich, Michael. 2003. Cyberscience. Research in the age of the internet. Wien: Austrian Academy of Sciences Press.

Nentwich, Michael and René König. 2012. Cyberscience 2.0. Research in the age of digital social networks. Frankfurt/New York: Campus.

NIH (National Institutes of Health). 2003. Final NIH statement on sharing research data. http://grants.nih.gov/grants/guide/notice-files/NOT-OD-03-032.html. Gesehen 01. November 2015.

OpenDOAR. 2015: Directory of Open Access Repositories. http://www.opendoar.org/. Gesehen 01. November 2015.

Parse. 2009. Insight into digital preservation of research output in Europe. http://www.parseinsight.eu/downloads/PARSE-Insight_D3-4_SurveyReport_final_hq.pdf. Gesehen 01. November 2015.

Ribes David and Charlotte P. Lee. 2010. Sociotechnical Studies of Cyberinfrastructure and eResearch: Current Themes and Future Trajectories. Computer Supported Cooperative Work 19: $231-244$.

Reichertz, Jo. 2015. Wie mit Daten umgehen? Persönlichkeitsrechte und Datenschutz in der Qualitativen Sozialforschung. Soziologie 44(2): 186-202.

Schimank, Uwe. 2012. Wissenschaft als gesellschaftliches Teilsystem. In Handbuch Wissenschaftssoziologie, Hrsg. Sabine Maasen, 113-123. Wiesbaden: Springer.

Star, Susan Leigh. 1999. The Ethnography of Infrastructure. American Behavioral Scientist 43: 377-391.

Star, Susan Leigh und Karen Ruhleder. 1996. Steps Towards an Ecology of Infrastructure: Design and Access for Large Information Spaces. Information Systems Research 7(1): 111134.

Stichweh, Rudolf. 1979. Differenzierung der Wissenschaft. Zeitschrift für Soziologie 8(1): 82101.

Suber, Peter. 2009. Timeline of the Open Access Movement. http://legacy.earlham.edu/ peters/fos/timeline.htm. Gesehen 01. November 2015. 
Taubert, Niels und Kevin Schön 2014: Online-Konsultation „Publikationssystem” Dokumentation und Auswertung. Berlin: BBAW. Persistent Identifier: urn:nbn:de:kobv:b4opus-26293.

Taubert, Niels. 2012. Online Editorial Management Systeme und die Produktion wissenschaftlicher Fachzeitschriften. Leviathan 40(2): 297-319.

Taubert, Niels. 2016. Formale wissenschaftliche Kommunikation. In Forschungsfeld Wissenschaftskommunikation. Hrsg. von Heinz Bonfadelli, Birte Fähnrich, Corinna Lüthje, Jutta Milde, Markus Rhomberg und Mike Schäfer. Wiesbaden: Springer-VS. (im Erscheinen).

Universität Heidelberg. (o. Jg.). Research Data Policy. http://www.uniheidelberg.de/universitaet/profil/researchdata. Gesehen 02. November 2015.

Volkmann, Ute, Uwe Schimank und Markus Rost. 2014. Two Worlds of Academic

Publishing: Chemistry and German Sociology in Comparison. Minerva 52(2): 187-212.

Ware, Mark. 2008. Peer review in scholarly Journals: Perspectives of the scholarly communication - an international study. Bristol: Mark Ware Consulting. http://www.publishingresearch.net/documents/PeerReviewFullPRCReport-final.pdf. Gesehen 01. November 2015.

Wellcome Trust. 2010. Policy on data management and sharing. http://www.wellcome.ac.uk/about-us/policy/policy-and-position-statements/wtx035043.htm. Gesehen 01. November 2015.

Wenger, Marc, François Ochsenbein, Daniel Egret, Pascal Dubois, François Bonnarel, Suzanne Borde, Françoise Genova, Gérard Jasniewicz, Suzanne Laloë, Soizick Lesteven, and Richard Monier. 2000. The SIMBAD astronomical database The CDS Reference Database for Astronomical Objects. Astronomy and Astrophysics 143(4): 9-22.

Whitley, Richard D. 1968. The formal communication system of science: A study of the organisation of British social science journals. The Sociological Review 16 (1): 162-179.

Wissenschaftsrat. 2011a. Übergreifende Empfehlungen zu Informationsinfrastrukturen. Berlin: Drs. 10466-11.

Wissenschaftsrat. 2011b. Empfehlungen zur Zukunft des bibliothekarischen Verbundsystems in Deutschland. 28. Berlin: Drs. 10463-11. 
Wouters, Paul. 2006. What is the matter with e-Science? - thinking aloud about informatisation in knowledge creation. http://www.pantaneto.co.uk/issue23/wouters.htm. Gesehen 01. November 2015. 